\title{
ALGUNOS ASPECTOS ECOLÓGICOS Y FITOGEOGRÁFICOS DE LAS ESPECIES DE CYPERACEAE EN EL VALLE DE MÉXICO. I. AFINIDADES ECOLÓGICAS*
}

\author{
SOCORRO GONZÁLEZ E.**,*** y J. RZEDOWSKI**,****
}

\section{RESUMEN}

Las ciperáceas se encuentran ampliamente distribuidas en el Valle de México, en prácticamente todos los tipos de vegetación, pero principalmente en lugares de suelo húmedo o inundado dentro de bosques de encino, pastizales, bosques de pino, matorrales xerófilos y bosques de Abies, entre los 2230 y 4100 m de altitud. Hay varias especies francamente acuáticas, por ejemplo todas las del género Scirpus, algunas de Eleocharis y unas pocas de Cyperus y Carex, pero también hay otras adaptadas a condiciones de poca humedad. El grueso de las especies ajusta la fenología de su reproducción a la época lluviosa; sólo algunas de las manifiestamente hidrófitås pueden encontrarse en flor durante la mayor parte del año.

\begin{abstract}
The Cyperaceae are widely distributed in the Valley of Mexico, practically in all vegetation types, but mainly in wet or inundated soil within oak forests, grasslands, pine forests, xerophilous scrubs and fir forests, in an altitudinal range of 2230 to $4100 \mathrm{~m}$. Several are evident aquatics, like all the species of Scirpus, some of Eleocharis as well as a few of Cyperus and Carex, but there are also other adapted to conditions of reduced humidity. The reproductive phenology of most of the species is linked with the rainy season; only some of the evident aquatics can be found in flower almost the year round.
\end{abstract}

*Trabajo parcialmente subvencionado por el Consejo Nacional de Ciencia y Tecnología, en el marco del proyecto "Flora y vegetación del Valle de México". Recibido para publicación en junio de 1983.

** Laboratorio de Botánica Fanerogámica, Escuela Nacional de Ciencias Biológicas, Instituto Politécnico Nacional, México 17, D.F.

***Dirección actual: CIIDIR-IPN Unidad Durango, Hidalgo 120, Vicente Guerrero, Dgo.

****Becario de la COFAA del Instituto Politécnico Nacional.

González-E S, Rzedowski J. 1983. Algunos aspectos ecológicos y fitogeográficos de las especies de Cyperaceae en el Valle de México. I. Afinidades ecológicas. Boletín de la Sociedad Botánica de México 45: 39-47. 


\section{INTRODUCCIÓN}

El estudio taxonómico de la familia Cyperaceae dentro del proyecto "Flora y vegetación del Valle de México" dio por resultado la detección de la existencia de 9 géneros, 79 especies y 4 variedades, dentro del área estudiada.

El Valle de México se localiza en el extremo meridional de la altiplanicie mexicana, en la porción central del país. Cuenta con una superficie aproximada de $7500 \mathrm{~km}^{2}$, y sus extremos de altitud sobre el nivel del mar oscilan entre 2230 y $5452 \mathrm{~m}$.

Los principales tipos de vegetación en el Valle son los siguientes: bosque de Abies, bosque mesófilo de montaña, bosque de Quercus, bosque de Pinus, matorral de Quercus, pastizales, matorral xerófilo, vegetaciór halófila y vegetación acuática y subacuática.

Para una descripción más extensa de las características del Valle de México puede consultarse el trabajo de uno de los autores (Rzedowski, 1979) en el Vol. I de la Flora fanerogámica del Valle de México.

\section{Generalidades}

La familia Cyperaceae se encuentra ampliamente distribuida en el Valle, en prácticamente todos los tipos de vegetación, desde los 2230 hasta los 4100 m de altitud. Tiene relativamente poca importancia ecológica dentro de la región, ya que muchas de sus especies están representadas por un escaso número de individuos; en cambio, florísticamente ocupa uno de los primeros lugares, siendo superada en número de especies por pocas familias.

Las ciperáceas se localizan por lo general en lugares de suelo húmedo o inundado, aunque varias especies están bien adaptadas a condiciones de poca humedad, como por ejemplo: Bulbostylis juncoides, B. funckii, Cyperus manimae, C. spectabilis, Cyperus sp. y Abildgaardia mexicana. Por otro lado, hay varias especies que se comportan como francamente acuáticas o subacuáticas: Cyperus laevigatus, C. niger, C. pycnostachyus, C. semiochraceus, Eleocharis bonariensis, E. densa, E. macrostachya, Scirpus americanus, S. maritimus var. paludosus, $S$. californicus, $S$. nevadensis y $S$. validus, así como algunas poblaciones de Carex praegracilis, Eleocharis acicularis y E. montevidensis. Otras especies son también comunes en lugares inundados, pero en condiciones ecológicas más o menos específicas, por ejemplo Carex brachycalama y C. kelloggii en medio de pinares y zacatonales subalpinos.

Scirpus y Eleocharis son los géneros que muestran mayor dependencia con respecto a la humedad del suelo, mientras que Bulbostylis, Abildgaardia y Fimbristylis son aparentemente los menos ligados al agua.

Algunas de las plantas anteriormente mencionadas, como $C$. niger y $E$. acicularis, son frecuentes en lugares encharcados dentro de varios tipos de vegetación entre los 2250 y $3500 \mathrm{~m}$ de altitud, pero otras, por ejemplo C. pycnostachyus, C. semiochraceus, $C$. virens, $E$. bonariensis, $S$. nevadensis y $S$. maritimus var. paludosus se encuentran restringidas a orillas de lagunas y canales en las partes bajas del Valle, y están en serio peligro de desaparecer de esta región debido a la desecación de sus hábitats; de hecho C. pycnostachyus y $E$. bonariensis han sido citadas para el Valle de diversas localidades donde ahora no se encuentran, y el escaso número de ejemplares revisados da idea de que estas especies se han visto bastante afectadas por la modificación de su medio ambiente.

Varias especies se desarrollan bien en lugares salobres localizados en las partes bajas del Valle: Scirpus maritimus var. paludosus y S. nevadensis pueden considerarse como 


\section{ECOLOGÍA Y FITOGEOGRAFÍA DE CYPERACEAE}

halófilas, ya que en el área se localizan exclusivamente en los alrededores del lago de Texcoco, donde se presenta el mayor grado de salinidad y alcalinidad.

Carex praegracilis, Cyperus laevigatus, $C$. pycnostachyus y $C$. semiochraceus se han colectado en el lago de Texcoco y en otras pocas localidades, mientras que Eleocharis macrostachya y $E$. montevidensis se conocen de dicha área pero están ampliamente distribuidas, pudiéndose considerar como halófilas facultativas. Cyperus esculentus es también tolerante a condiciones de salinidad.

La mayor parte de los miembros de esta familia prefieren lugares abiertos y asoleados; las que crecen en bosques y matorrales son más abundantes en los claros y partes menos densas dentro de estos tipos de vegetación, excepto unas pocas especies de Carex que crecen en cañadas.

\section{Distribución en los principales tipos de vegetación del Valle de México}

En la tabla 1 se resume el número de especies y variedades de cada género presentes en los principales tipos de vegetación del Valle, así como el porcentaje con respecto del total de especies encontradas en este trabajo.

El género Cyperus es el único representado en todos los tipos de vegetación, aunque alcanza su mayor diversificación en pastizales, bosques de encino, matorrales xerófilos y bosques de pino (en ese orden). Este género es el más abundante en el Valle, tanto respecto del número de taxa como del número de individuos, siendo además el de mayor tolerancia ecológica. Se localiza entre los 2230 y 3250 m de altitud, tanto en lugares de suelo relativamente seco como en terrenos inundados. Algunas especies son comunes en varios tipos de vegetación, por ejemplo Cyperus manimae, C. spectabilis, C. seslerioides, $C$. hermaphroditus y $C$. huarmensis, las que muestran además una fuerte variación morfológica, especialmente notable en las dos primeras, en las que la gran diversidad de formas observadas no parece tener una relación directa con el hábitat. Por el contrario, en $C$. seslerioides y $C$. niger se encontró que las plantas varían de acuerdo al ambiente, ya que su tamaño se reduce a medida que aumenta la altitud del lugar donde crecen, encontrándose plantas enanas en localidades a más de $3000 \mathrm{~m}$ de altitud, las cuales pueden ser consideradas como ecofenos alpinos.

Otras especies son bastante escasas y se conocen sólo de una o muy pocas localidades: C. aschenbornianus, C. flavescens var. piceus, C. mutisii, C. odoratus, C. pycnostachyus, C. rotundus, $C$. virens y $C$. arsenii, siendo probable que esta última haya desaparecido en la actualidad del Valle.

Cyperus es además el único género del Valle con especies que se comportan como malezas: C. esculentus, principalmente como arvense, y $C$. spectabilis, $C$. manimae y $C$. hermaphroditus como ruderales y en ocasiones arvenses en forma facultativa, aunque estas tres especies se desarrollan más bien en hábitats naturales. C. rotundus es una importante maleza arvense en regiones calientes de todo el mundo, pero para el Valle de México se conoce sólo de una colecta, como maleza en un jardín y debe ser considerada más bien como una introducción temporal.

Carex se encuentra también en casi todos los tipos de vegetación, en altitudes que fluctúan entre 2250 y $4100 \mathrm{~m}$. Alcanza su mayor diversificación en los bosques de pino, bosques de Abies, bosques de encino y en la vegetación alpina y subalpina. En general, los miembros de este género son de lugares húmedos, pero no se comportan como acuáticas o subacuáticas, excepto $C$. praegracilis, y algunas poblaciones de $C$. brachycalama, C. angustior, C. kelloggii, C. peucophila y $C$. volcanica que se desarrollan en lugares inundados o de suelo muy húmedo. 
TABLA 1. Número de especies y variedades de Ciperáceas presentes en varios tipos de vegetación* del Valle de México y porcentaje con respecto del total de especies y variedades localizadas en el Valle $a$

\begin{tabular}{|c|c|c|c|c|c|c|c|c|c|c|c|c|c|c|c|c|c|c|c|c|c|}
\hline & \multicolumn{2}{|c|}{$B A$} & \multicolumn{2}{|r|}{$B M$} & \multicolumn{2}{|c|}{$B P$} & \multicolumn{2}{|c|}{$B E$} & \multicolumn{2}{|r|}{$B J$} & \multicolumn{2}{|c|}{$M E$} & \multicolumn{2}{|r|}{$P$} & \multicolumn{2}{|c|}{$Z A$} & $M X$ & \multicolumn{2}{|c|}{$A S$} & \multirow{2}{*}{\multicolumn{2}{|c|}{$M$}} \\
\hline Abildgaardia & & & & & & & & & & & & & 1 & 100 & & & 1100 & & & & \\
\hline Bulbostylis & & & & & 1 & 50 & 1 & 50 & 1 & 50 & 1 & 50 & 2 & 100 & & & 150 & & & & \\
\hline Carex & 9 & 32 & 5 & 17.9 & 14 & 50 & 8 & 28.6 & 1 & 3.6 & 2 & 7.1 & 4 & 14.3 & 8 & 29 & & 1 & 3.6 & & \\
\hline Cyperus & 3 & 9.7 & 1 & 3.2 & & 2.3 & & 48.4 & 1 & 3.2 & 1 & 3.2 & & 51.6 & 2 & 6.5 & ${ }^{11} 35.5$ & 5 & 16.1 & 5 & 16.1 \\
\hline Eleocharis & 2 & 25 & 1 & 12.5 & 2 & 25 & 4 & 50 & 1 & 12.5 & 1 & 12.5 & 3 & 37.5 & & & $2 \quad 25$ & 5 & 62.5 & & \\
\hline Fimbristylis & & & & & & & & 66.6 & & & & & & 66.6 & & & 133.3 & & & & \\
\hline Rhynchospora & 1 & 50 & & & 1 & 50 & 2 & 100 & & & & & 2 & 100 & & & & & & & \\
\hline Scirpus & & & & & & & & & & & & & & & & & & 5 & 100 & & \\
\hline Uncinia & 1 & 50 & 2 & 100 & 1 & 50 & & & & & & & & & & & & & & & \\
\hline Total & & 19.5 & 9 & 11.0 & & 35.4 & & 39.0 & 4 & 4.9 & 5 & 6.1 & & 36.6 & & 12.2 & $16_{19.5}$ & & 19.5 & 5 & 6.1 \\
\hline
\end{tabular}

*Los tipos de vegetación considerados aquí son los siguientes: bosque de Abies (BA), bosque mesófilo de montaña (BM), bosque de Pinus (BP), bosque de Quercus (BE), bosque o matorral de Juniperus (BJ), matorral de Quercus (ME), pastizales (P), zacatonal alpino o subalpino (ZA), matorral xerófilo (MX), vegetación acuática y subacuática (AS) y medio arvense y ruderal (M). 


\section{ECOLOGÍA Y FITOGEOGRAFÍA DE CYPERACEAE}

Muchas de las especies de Carex forman pequeñas poblaciones restringidas por lo general a una o pocas localidades. De algunas se han encontrado únicamente individuos muy aislados entre sí, sobre todo en plantas de porte robusto y amacollado. El género Eleocharis ocupa el tercer lugar en amplitud de distribución dentro del Valle, aunque más de la mitad de sus especies son acuáticas o subacuáticas. Algunas de éstas, como $E$. montevidensis y $E$. macrostachya son abundantes y muy variables morfológicamente, mientras que otras, como $E$. bonariensis y $E$. cancellata son muy escasas.

Bulbostylis y Abildgaardia están mucho mejor representados en los pastizales y, con menos frecuencia, en matorrales xerófilos, aunque $B$. juncoides se presenta también en otros tipos de vegetación. Abildgaardia mexicana es subdominante en algunos pastizales ubicados en la parte occidental del Valle.

Rhynchospora y Fimbristylis se presentan principalmente en pastizales y bosques de encino. En Rhynchospora kunthii se observó también un fenómeno ya mencionado para dos especies de Cyperus: las plantas que crecen en lugares a $3000 \mathrm{~m}$ o más de altitud son de porte bajo, y presentan además hojas cortas e inflorescencias pequeñas y más o menos espiciformes, a diferencia de las plantas altas y con corimbos bien espaciados típicas de lugares más bajos.

Las dos especies de Uncinia del Valle se localizan en el bosque mesófilo de montaña, aunque $U$. phleoides se ha colectado también en el bosque de Abies y en un bosque perturbado de Pinus, entre los 2700 a 3000 m de altitud.

Scirpus es el único género en el que todas las especies son exclusivas de un tipo de hábitat, ya que dichas plantas son acuáticas. Cuatro de ellas crecen a la orilla de canales y lagunas entre 2240 y 2260 m de altitud, en la parte más baja del Valle, y solamente $S$. californicus "sube" hasta los $2600 \mathrm{~m}$.

3. Participación de las subfamilias de la familia Cyperaceae en diferentes comunidades vegetales del Valle de México

En la Fig. 1 se ilustra la participación diferencial de las tres subfamilias presentes en el Valle de México, en diferentes comunidades vegetales dentro del Valle. Las comunidades se distribuyeron en la figura de acuerdo a la altitud y tipo de clima que les corresponde.

La subfamilia Scirpoideae (Abildgaardia, Bulbostylis, Cyperus, Eleocharis, Fimbristylis y Scirpus) es la mejor representada en el Valle, tanto en número de especies como de individuos, así como la más ampliamente distribuida, aunque es más abundante en pastizales, bosque de encino, matorral xerófilo, vegetación acuática y bosques de pino. Se localiza desde las partes más bajas del Valle $(2230 \mathrm{~m}$ ) hasta los $3500 \mathrm{~m}$.

La subfamilia Caricoideae (Carex y Uncinia) prospera en lugares más bien fríos, entre 2250 y $4100 \mathrm{~m}$ de altitud. Se manifiesta como dominante en los zacatonales alpinos y subalpinos, bosque mesófilo, bosque de oyamel y bosques de pino.

Rhynchosporoideae es la menos favorecida por las condiciones ecológicas del Valle, contando con únicamente dos especies que prosperan entre 2300 y $3150 \mathrm{~m}$ de altitud, principalmente en pastizales y bosques de encino.

\section{Fenología}

En la tabla 2 se presenta un resumen de las épocas en que las ciperáceas del Valle se encuentran en flor y en fruto, con base en los especímenes de herbario revisados. 


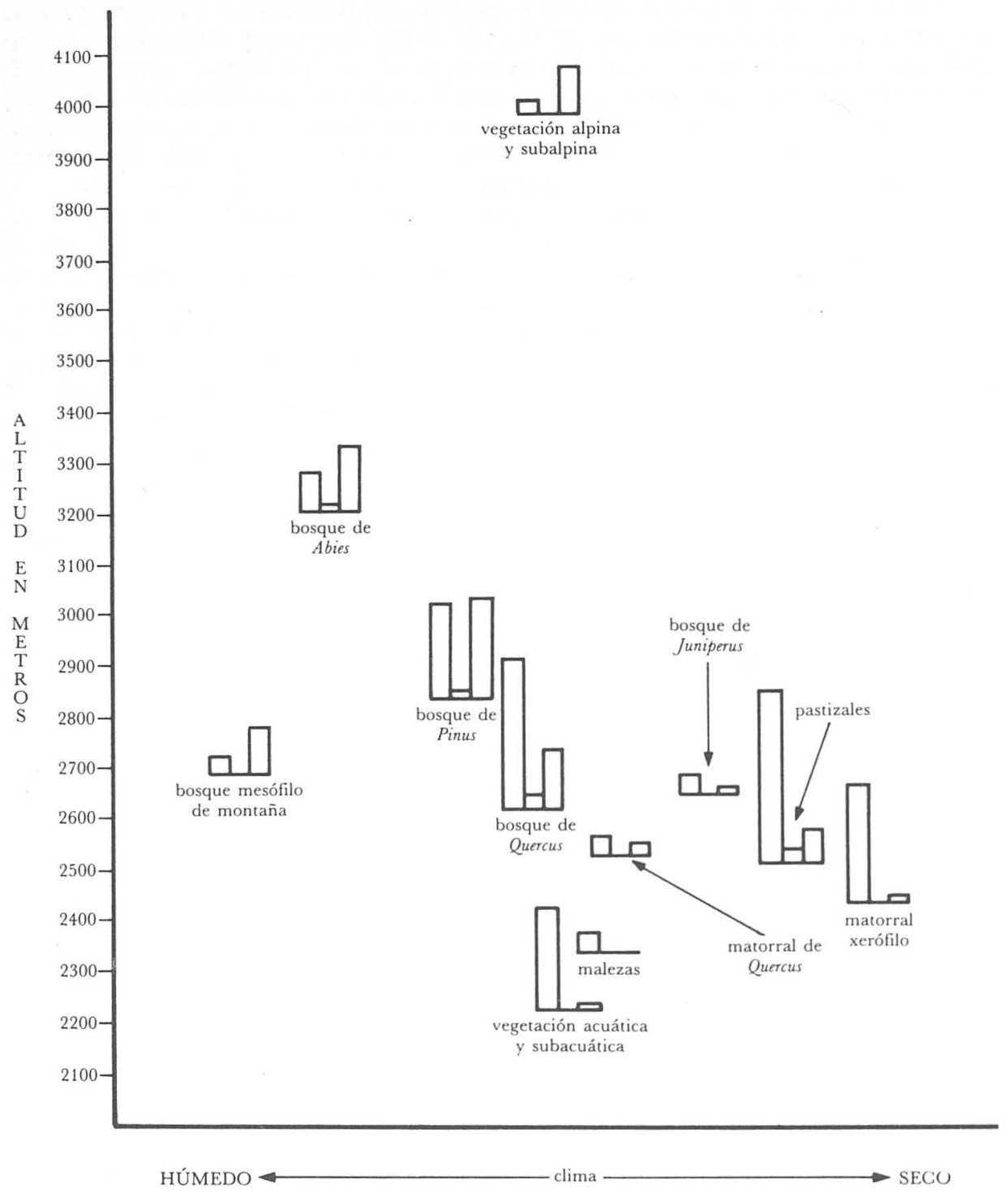

Fig. 1. Participación diferencial de 3 subfamilias de la familia Cyperaceae en diferentes comunidades vegetales del Valle de México, calculada a nivel de especie. Las subfamilias se representan por medio de columnas, de izquierda a derecha: Scirpoideae, Rhynchosporoideae y Caricoideae. La altura de cada columna está en relación directa con el número de especies de la subfamilia que se conoce para cada tipo de vegetación. 
TABLA 2. Épocas de floración y fructificación de las especies de Cyperaceae del Valle de México, con base en los ejemplares de herbario revisados durante este trabajo: Flor $\bigcirc$; Fruto + ; Flor y fruto $\oplus$

\begin{tabular}{|c|c|c|c|c|c|c|c|c|c|c|c|c|}
\hline & Ene & $\mathrm{Feb}$ & Mar & $A b r$ & May & Jun & Jul & Ago & Sep & Oct & Nov & Dic \\
\hline $\begin{array}{l}\text { Abildgaardia mexicana } \\
\text { Bulbostylis funckii }\end{array}$ & & & & & & & $0+$ & $\begin{array}{c}++ \\
+\end{array}$ & $\begin{array}{c}+++ \\
+\end{array}$ & +++ & & \\
\hline B. juncoides & & & & & & D & $0++$ & +++ & +++ & +++ & + & \\
\hline Carex angustior & & & & ++ & & ++ & +++ & +++ & ++ & ++ & & + \\
\hline C. athrostachya & & & & & & & & & & & + & + \\
\hline C. boliviensis & & & & & & + & ++ & $0+$ & & + & & \\
\hline $\begin{array}{l}\text { C. brachycalama } \\
\text { C. chordalis }\end{array}$ & & & & 0 & 0 & & +++ & & & & & \\
\hline C. conspecta & & & & & & & + & & & + & + & \\
\hline C. cortesii & & & 0 & ++ & 0 & & & & & & & \\
\hline $\begin{array}{l}\text { C. coulteri } \\
\text { C. egglestonii }\end{array}$ & & & & & & ++ & $\begin{array}{c}\mathrm{O}+ \\
+\end{array}$ & ++ & +++ & + & + & \\
\hline C. geophila & & & & & & & & & + & & & \\
\hline C. humboldtiana & & & & & & & & & +++ & & + & \\
\hline C. involucratella & & & & & & & + & & & & & \\
\hline C. kelloggii & & & & & & & $\oplus \oplus$ & & & & + & + \\
\hline C. longicaulis & & & & & & $O++$ & $\oplus++$ & +++ & +++ & ++ & + & \\
\hline $\begin{array}{l}C . \text { longii } \\
C . \text { marianensis }\end{array}$ & & & & & $\oplus$ & & + & $\oplus++$ & ++ & & & + \\
\hline C. peucophila & & & & 0 & & $\bigcirc \oplus \oplus$ & $\oplus++$ & +++ & +++ & ++ & & \\
\hline C. planostachys & & & & & & 0 & & & & & + & \\
\hline C. polystachya & & & & & & & & & & & + & \\
\hline C. praegracilis & & & & 00 & & $0++$ & 0 & & & & & \\
\hline C. psilocarpa & & & & + & 0 & & & & + & + & + & + \\
\hline C. townsendii & & & & & & & $0++$ & & + & & & \\
\hline C. tuberculata & & & & $\bigcirc \oplus$ & + & +++ & +++ & + & & ++ & ++ & \\
\hline C. turbinata & & & & & & & & +++ & & & & \\
\hline C. vallicola & & & & & & & & $\oplus$ & + & & & \\
\hline C. volcanica & ++ & & & $\oplus \oplus$ & & & $(\oplus++$ & +++ & +++ & + & + & + \\
\hline C. xalapensis & & & & & & & ++ & ++ & & + & + & \\
\hline Carex sp. & & & & & & & $\infty^{\circ}$ & & & + & & \\
\hline Cyperus apiculatus & & & & & & & & & ++ & + & & \\
\hline C. aristatus & & & & & & & & & +++ & +++ & & \\
\hline $\begin{array}{l}\text { C. arsenii } \\
\text { C. aschenbornianus }\end{array}$ & & & & & & & & + & & & & \\
\hline $\begin{array}{l}\text { C. aschenbornianus } \\
\text { C. esculentus }\end{array}$ & & & & & & $\Omega \Omega$ & $\Omega 0$ & & $\rightarrow \oplus+$ & $\oplus+$ & & \\
\hline C. fendlerianus & & & & & & & & $\begin{array}{l}\mathcal{U}++ \\
+++\end{array}$ & $\begin{array}{l}\oplus \oplus+ \\
+++\end{array}$ & $(\oplus)+$ & ++ & \\
\hline C. flavescens & & & & & & & & & +++ & ++ & + & \\
\hline
\end{tabular}




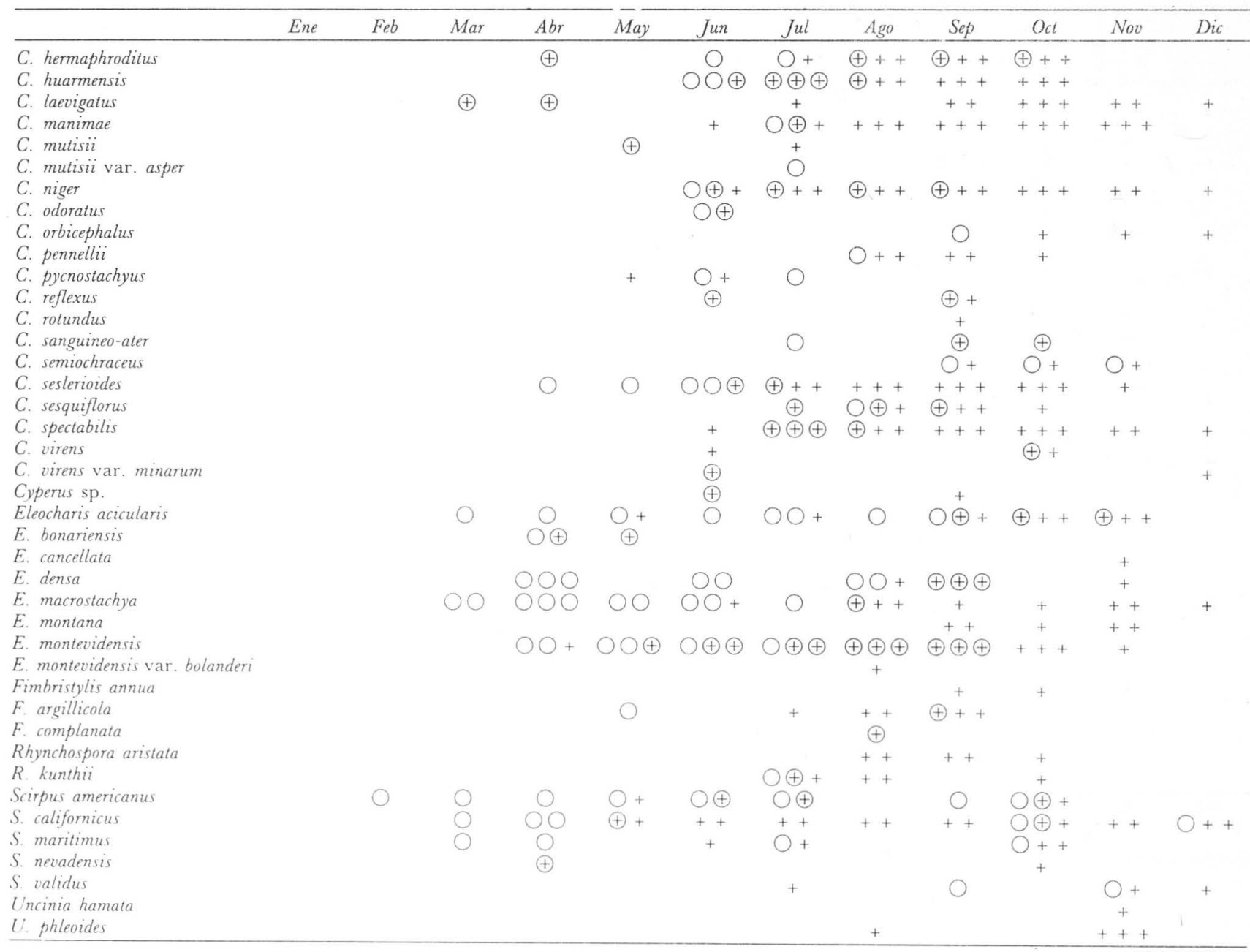


Esta información es útil, ya que para facilitar el proceso de identificación es de suma importancia que el material se encuentre en plena madurez.

En general, la época de fructificación está comprendida entre julio y octubre, extendiéndose hasta diciembre en muchos casos. La floración se inicia en abril, aunque varias especies acuáticas se han colectado en flor en marzo e incluso una desde febrero, reflejando la relativa independencia que tienen las plantas acuáticas de las condiciones climáticas, ya que la precipitación pluvial no es un factor limitante para su desarrollo. Además, esas plantas se encuentran en las partes más bajas del Valle, en donde las temperaturas bajas de invierno se retiran antes que en lugares situados a mayor altitud.

Por otro lado, dos especies distribuidas desde Alaska hasta México (Carex athrostachya y C. kelloggii) presentan un retardo en su época de fructificación, reflejando en apariencia una adaptación a las bajas temperaturas.

\section{BIBLIOGRAFIA}

RZEDOWSKI, J. Capítulos introductorios, en: RZEDOWSKI, J. y G.C. DE RZEDOWSKI (Eds.). 1979. Flora fanerogámica del Valle de México, Vol. I. Compañía Editorial Continental, México. 